\title{
Investigation of chromosome $2 q$ in osteoarthritis of the hand: no significant linkage in a Tasmanian population
}

\author{
J Stankovich, M M Sale, H M Cooley, M Bahlo, A Reilly, J L Dickinson, G Jones
}

Ann Rheum Dis 2002;61:1081-1084

See end of article for authors' affiliations

Correspondence to:

Dr J Stankovich, Menzies

Centre for Population

Health Research, University

of Tasmania, GPO Box

252-23, Hobart, Tasmania

7001, Australia;

Jim.Stankovich@utas.edu.au

Australia

Accepted 26 April 2002
Background: Previous studies have suggested a strong genetic component to osteoarthritis (OA), especially that of the hand, and three linkage studies have suggested the existence of susceptibility loci in disparate regions of chromosome $2 \mathrm{q}$.

Objective: To examine for linkage to $2 q$ in a Tasmanian population of women and men with familial hand OA.

Methods: Hand OA (distal interphalangeal, carpometacarpal, and Heberden's nodes) was assessed by a combination of hand photographs and radiographs. A non-parametric linkage (NPL) analysis was performed on chromosome $2 q$ of 69 members in 22 families with severe distal interphalangeal joint $\mathrm{OA}$ using Genehunter. A quantitative trait linkage analysis of a larger group of 456 members in 68 families was also performed using SOLAR.

Results: The maximum non-parametric linkage score was $1.05(p=0.15)$ at marker ILIR 1, close to the centromere. All components of hand OA scores had significant heritability in this dataset $28 \%-35 \%$, all $p<0.001$ ). Despite this, the quantitative trait analysis (after adjustment for age and, where appropriate, sex) yielded maximum LOD scores of 0.90 for Heberden's nodes (both sexes combined), and 1.19 for carpometacarpal OA score (women only).

Conclusions: These results do not provide confirmation of linkage on chromosome $2 q$ in the larger white population with hand $O A$. They suggest that there are regional variations in the genetic cause of hand $O A$ and that other loci not on $2 q$ may be important in this disease.
O steoarthritis (OA) is the leading cause of musculoskeletal disability in western countries. ${ }^{1}$ Although knee and hip OA cause the greatest disability, hand OA also results in disability. ${ }^{2}$ The prevalence of hand OA in the community is very high, with population based estimates ranging from $30 \%$ to $52 \%{ }^{3-5}$ and is strongly age dependent, ranging in one survey from $5 \%$ in subjects aged $18-39$ to $72 \%$ in subjects aged 60 and over. ${ }^{6}$

Some studies have suggested that both genetic and environmental factors contribute to the development of OA, with different risk factors at different joints, and different risk factors for men and women. ${ }^{7}$ There is recent evidence to suggest that the heritability of hand OA and hip OA may be higher than the heritability of knee OA..$^{8-10}$ In addition, some reports suggest that the genetic component of OA may be greater for women than for men. ${ }^{11}{ }^{12}$ There has been weak evidence for the association of various environmental factors with hand OA.

Three previous studies have identified chromosome $2 \mathrm{q}$ as one of the regions of the human genome most likely to harbour genes for OA, although at divergent regions of this chromatid. Two of these linkage studies investigated hand $\mathrm{OA},{ }^{13}{ }^{14}$ whereas the third was a study of hip and knee OA. ${ }^{15}$

Taken together, these three studies suggest that there may be OA susceptibility genes on chromosome 2q. The aim of this study, therefore, was to examine for the presence of linkage to $2 \mathrm{q}$ in a Tasmanian population of men and women with familial hand OA.

\section{METHODS}

Tasmania is an island state of Australia. Its population of 472000 is predominantly white, ${ }^{16}$ and is similar genetically to other Anglo-Celtic populations, although it is more homogeneous than some other populations. ${ }^{17}$ At the time of the study, statewide rheumatology specialist services in Tasmania were provided by three rheumatologists in a single practice based in the capital city of Hobart. Subjects were recruited from the records of this practice. All patients who had a doctor's diagnosis of hand OA and at least one living relative with hand OA were invited to take part, along with their affected and unaffected relatives. Ethical approval was granted for the study by the Royal Hobart Hospital human research ethics committee and all participants provided informed written consent. Participants underwent a comprehensive protocol involving collection of blood, as well as detailed assessment of hand OA, anthropometrics, environmental factors, pain, and function.

Presence of hand OA was assessed using two methods. Radiographic disease was assessed using an atlas ${ }^{18}$ for joint space narrowing and osteophytes at the distal interphalangeal (DIP) and first carpometacarpal (CMC) joints from a single anteroposterior radiograph of the hands, performed according to a standardised protocol by the same two investigators (HMC, GJ) simultaneously, with a single score obtained by consensus. The scores for each component at each joint could vary from 0 (no disease) to 3 (severe disease). Scores for DIP disease could vary from 0-48 and CMC disease from 0-12.

A photograph of both hands was taken with an Elicar Medical Macro MS2 camera. Photographs were then scored as to the presence ( 1 ) or absence $(0)$ of Heberden's nodes in each DIP joint by two investigators (GJ, HMC) simultaneously, with a single score obtained by consensus without reference to radiographs. Heberden's node scores could vary from $0-8$.

The reproducibility of all measures was assessed by rescoring widely varying samples of 45 radiographs and 50

Abbreviations: $C M$, centimorgans; CMC, first carpometacarpal; DIP, distal interphalangeal; MLS, multipoint LOD score; NPL, non-parametric linkage; $\mathrm{OA}$, osteoarthritis 


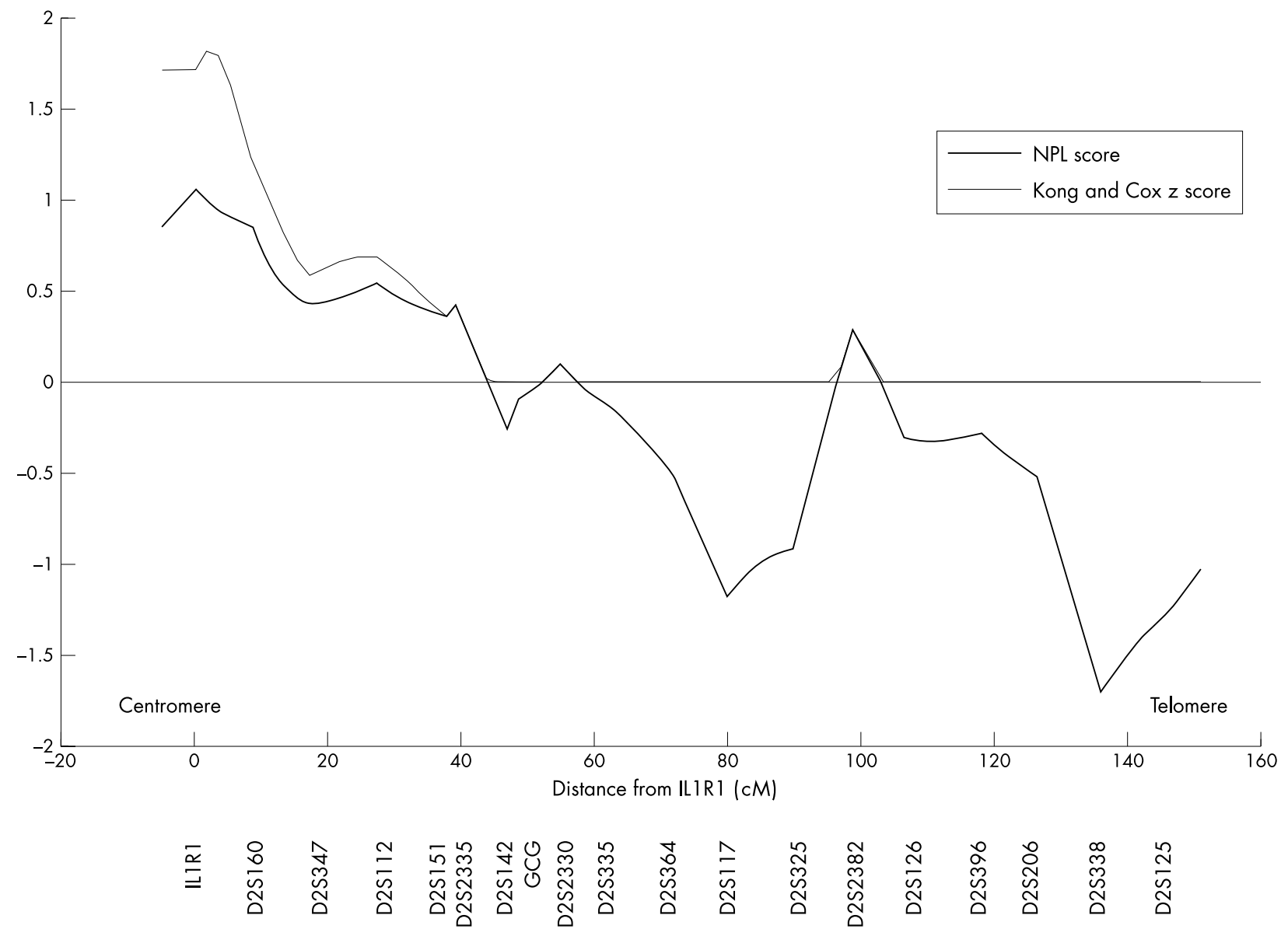

Figure 1 Results of the dichotomous trait analysis. A plot of the NPL score and the Kong and Cox z score along chromosome $2 q$ for 69 affected subjects in 22 families. Locations of the 19 microsatellites genotyped are indicated, and distance is shown in centiMorgans (cM).

photographs one week after they were first scored. All intraclass correlations were at least $0.94 .^{2}$ All phenotyped subjects, plus an additional 77 spouses, were genotyped using fluorescent automated fragment analysis technology (Applied Biosystems ABI PRISM 377 DNA Sequencers) at the Australian Genome Research Facility, Melbourne, using 19 microsatellite markers from chromosome 2q. These included the 16 markers for 2q from Applied Biosystems ABI PRISM Linkage Mapping set MD-10, and three additional microsatellite markers (IL1R1, D2S2335, and GCG) reported to be in linked regions in previous studies. ${ }^{13}{ }^{14}$ Genetic distances between the ABI markers were taken from the Marshfield chromosome 2 sex averaged linkage map (http:// research.marshfieldclinic.org/genetics/), and the positions of the additional markers were estimated using the same map in combination with the orderings of Whitehead contigs WC2.8 and WC2.11 (accessed via the Genome Database, http:// www.gdb.org), physical map distances from the genome browser of the National Center for Biotechnology Information (http://www.ncbi.nlm.nih.gov), and figure 2 in a previous OA linkage study. ${ }^{14}$ Pedcheck ${ }^{19}$ was used to detect genotyping and pedigree errors.

Both dichotomous trait and quantitative trait analyses were used to test for linkage. For a dichotomous trait, affected patients only analysis, families and individual members were only included if they satisfied identical severity and similar age criteria to those used in a previous Finnish study. ${ }^{14}$ This was done so that results between the two studies could be directly compared. Families were included if they contained a proband 55 years old or younger with at least one non-zero Altman atlas DIP joint osteophyte score in each hand, and a DIP joint osteophyte score of 2 or 3 in at least one joint (equiv- alent to third or fourth degree radiographic OA respectively on the Kellgren-Lawrence scale ${ }^{20}$ used in the Finnish study). Relatives were classed as "affected" either if they were the sibling of a proband and had at least one non-zero DIP joint osteophyte score in each hand (at least second degree radiographic OA on the Kellgren-Lawrence scale), or if they were 55 or younger and had at least one non-zero DIP joint osteophyte score in each hand. Non-parametric linkage (NPL) scores were calculated using Genehunter $2^{2122}$ with the $S_{\text {pairs }}$ statistic, and Genehunter-Plus was used to calculate the less conservative Kong and Cox z score allowing for incomplete inheritance information. ${ }^{23}$ Non-parametric scores and z scores from each pedigree were combined by using the default weighting with scores from all pedigrees weighted equally. All pedigrees were small enough to include all affected persons in a full multipoint analysis. Of the unaffected relatives and older parents who had been genotyped, as many as possible were included in the multipoint analysis (within the constraints on pedigree size imposed by Genehunter) to improve the accuracy of haplotype reconstruction.

Several parametric analyses were also performed using the same criteria outlined above to define affected subjects. For these analyses the remaining subjects had to be classed as unaffected or unknown. Subjects 50 or older without DIP joint osteophytes in both hands were classed as unaffected; all other subjects were classed as unknown (those younger than 50 with insufficient abnormalities to be classed as affected, and those older than 55 with sufficient abnormalities to prevent them being classed as unaffected).For the parametric calculations, Genehunter 2 was used for a multipoint analysis and extra unaffected relatives and older parents were included in two point analyses with Fastlink version 4.1. ${ }^{24}$ 
Table 1 Characteristics of the study population (456 subjects)

\begin{tabular}{ll}
\hline & Mean (SD) \\
\hline Age (years) & $55.2(14.7)$ \\
Height $(\mathrm{cm})$ & $164.7(9.9)$ \\
Weight $(\mathrm{kg})$ & $74.6(16.2)$ \\
Distal interphalangeal joint score & $12.0(13.4)$ \\
Carpometacarpal joint score & $3.1(3.6)$ \\
Heberden's node score & $2.7(3.0)$ \\
\hline
\end{tabular}

A multipoint variance components analysis-testing for the presence of an additive quantitative trait locus on $2 \mathrm{q}$-was carried out using SOLAR. ${ }^{25}$ Three trait scores were analysed: (a) the DIP joint radiographic score (possible range 0-48), (b) the CMC joint score (0-12), and (c) the Heberden's node score $(0-8)$. Each trait was analysed for all subjects combined, as well as for women and men separately. The software was first used to estimate heritability after adjusting for the covariates age, sex, and an agexsex interaction, and correcting for ascertainment by conditioning on the probands. Age stratification was not performed. Evidence for linkage was then assessed at various points on the chromosome by testing the null hypothesis that the additive genetic variance due to a quantitative trait locus at each point was zero. This test for linkage assumes that trait scores are normally distributed. However, simulations have suggested that non-normal trait distributions with a coefficient of kurtosis less than 2 do not yield excessive type I error rates. ${ }^{26}$

\section{RESULTS}

A total of 7116 genotypes were measured. Of these, 82 (1.2\%) were eliminated after checking for inheritance errors using Pedcheck.

Sixty nine subjects in 22 families were classed as affected using the dichotomous trait definition. The three largest families contained 10, seven, and five affected members; the other 19 families contained two to four affected members. There were 67 affected sibling pairs ( 38 independent affected sibling pairs), two affected avuncular pairs, and 39 affected cousin pairs. The affected subjects ( 44 women and 25 men) had a mean age of 53.2 (SD 6.2) years and ranged in age from 34 to 67 years. Twenty one of the affected subjects were graded with a maximum Altman DIP osteophyte score of 1 in any jointequivalent to second degree radiographic OA on the KellgrenLawrence scale. ${ }^{20}$ Of the remaining subjects, 29 had a maximum Altman score of 2, and 19 had a maximum score of 3.

The maximum NPL score was $1.05(\mathrm{p}=0.15)$ at marker ILIRl, close to the centromere (fig 1). The Kong and Cox $\mathrm{Z}$ score reached a maximum of $1.81(\mathrm{p}=0.035)$ at the same marker. There was a similar lack of evidence for linkage when several dominant and recessive parametric models were tested, using both Fastlink and Genehunter (including the dominant model for which suggestive linkage was reported in the Finnish study ${ }^{14}$; results not shown).

For the quantitative trait analysis, phenotypic data were available for a total of 456 members (295 women and 161 men) in 68 families, with two to 21 members per family. Some characteristics of these members are given in table 1. They ranged in age from 24 to 92 years. Radiographic DIP joint scores, CMC joint scores, and Heberden's node scores were non-zero for $63 \%, 53 \%$, and $51 \%$ of members respectively and ranged from 0 to their maximum values $(48,12$, and 8 respectively). Spearman's correlation was 0.73 between radiographic DIP scores and Heberden's node scores, 0.64 between radiographic DIP scores and CMC scores, and 0.50 between CMC scores and Heberden's node score (all p values $<0.001$ ). All scores were strongly associated with age and sex: age and sex and their interaction term explained $43 \%$ of the variance in DIP scores, $38 \%$ of the variance in CMC scores, and $31 \%$ of the variance in Heberden's node scores.

After adjusting for these covariates, the estimated heritabilities of the DIP, CMC, and Heberden's node scores were $35 \%, 35 \%$, and $28 \%$ respectively, and all differed significantly from zero $(p<0.001)$. The coefficients of kurtosis for the covariate adjusted scores were all less than 0.7.

There was no evidence of an additive quantitative trait analysis on $2 \mathrm{q}$ influencing any of the three traits (table 2). When women and men were analysed together, the maximum LOD score was 0.90 for Heberden's nodes at a locus $140 \mathrm{cM}$ distal to ILIRl. When women and men were analysed separately, the maximum LOD score across $2 \mathrm{q}$ for any analysis was 1.19 for CMC joints in women.

\section{DISCUSSION}

Despite a comprehensive approach with high quality phenotypic data, there was no evidence of linkage on chromosome $2 \mathrm{q}$ in the Tasmanian population to support previous linkage findings in other populations.

In an analysis of 99 siblings from 44 families in England affected with nodal OA, two point linkage (pointwise $p$ values $<0.05$ ) was reported for three microsatellite markers spanning approximately $70 \mathrm{cM}$ in the regions $2 \mathrm{q} 23-\mathrm{q} 35$ in the middle of $2 \mathrm{q} \cdot{ }^{13}$ In a Finnish study, a genomewide scan was conducted on 64 siblings from 27 sibships with severe osteophytes in their distal interphalangeal joints. ${ }^{14}$ Suggestive linkage was reported for $2 \mathrm{q}$, very close to the centromere (two point LOD score 2.34, $\left.\mathrm{p}=5.1 \times 10^{-4}\right)$. A second linkage peak was detected some $20 \mathrm{cM}$ distal (two point LOD score 1.48; $\left.\mathrm{p}=4.5 \times 10^{-3}\right)$. Multipoint analysis yielded $\mathrm{p}$ values of $1 \times 10^{-4}$ and $7 \times 10^{-4}$ respectively at these two loci.

\begin{tabular}{|lll|}
\hline Table 2 & Results of the quantitative trait analysis & \\
\hline Subjects & Quantitative trait (maximum value) & $\begin{array}{l}\text { Maximum multipoint LOD } \\
\text { score* (locus (cM)) }\end{array}$ \\
\hline All $(\mathrm{n}=456)$ & Distal interphalangeal radiographic score (48) & $0.80(140)$ \\
& Carpometacarpal radiographic score (12) & $0.61(146)$ \\
& Heberden's node score (8) & $0.90(140)$ \\
Women ( $\mathrm{n}=295)$ & Distal interphalangeal radiographic score (48) & $0.66(136)$ \\
& Carpometacarpal radiographic score (12) & $1.19(126)$ \\
& Heberden's node score (8) & $0.81(136)$ \\
Men (n=161) & Distal interphalangeal radiographic score (48) & $0.15(35)$ \\
& Carpometacarpal radiographic score (12) & $0.04(105)$ \\
& Heberden's node score (8) & $0.40(90)$ \\
\hline *Distances in centiMorgans (cM) measured distal to marker IL1R1. & \\
\hline
\end{tabular}


In a United Kingdom linkage study of knee and hip $\mathrm{OA}^{15}$ a genomewide scan was conducted on a much larger set of 481 families, each containing at least one sibling pair who had had hip or knee joint replacements. A multipoint LOD score (MLS) of 1.22 was reported at a locus between the linkage sites on $2 \mathrm{q}$ reported in the English nodal OA study.$^{13}$ This MLS increased to $2.19\left(\mathrm{p}=7.4 \times 10^{-4}\right)$ in the families concordant for hip only disease.

For our dichotomous trait analysis, we had a similar number of affected people, 69 , as in the Finnish study, ${ }^{14}$ which had 64, and a similar number of affected sibling pairs $(67 v$ $50)$. The same degree of DIP joint radiographic OA was required in both studies for subjects to be classed as affected, and the mean age of those affected was five years younger in our study ( 53.2 years $v 58.9$ years). However, our study failed to reproduce the suggestive linkages reported close to the centromere. Nevertheless, it is worth noting that the highest linkage peak identified in our analysis (NPL score of 1.05) occurred at one of the markers (ILIRl) where suggestive linkage was identified in the Finnish study. Our result may represent a minor contribution of this locus in a more heterogeneous population. However, larger sample sizes would be needed to investigate this hypothesis.

For most disease models the variance components analysis with 456 phenotypic scores will have greater power to detect linkage than the dichotomous trait analysis with 69 affected people. Using simulations and theoretical results for the power of variance components analysis ${ }^{27}{ }^{28}$ based on the total number of relative pairs in our sample (439 sibling pairs, 589 aunt pairs, 559 cousin pairs-including both concordant and discordant relative pairs), we have about $70 \%$ power to detect a LOD score of 2 at a quantitative trait locus with heritability of $30 \%$. However, in our variance components analysis, no loci reached this level of significance for any quantitative trait.

Considering that our sample size was comparable with or greater than sample sizes in previous hand OA studies reporting linkage, it seems likely that loci outside $2 \mathrm{q}$ contribute to susceptibility or severity of hand OA in the Tasmanian population, and loci in 2 q make at most a small contribution, indicating the need for genotyping in other regions of the genome.

\section{ACKNOWLEDGEMENTS}

We thank the families for their participation. Thanks also go to Sr Catrina Boon who coordinated fieldwork for this study and Michele Brown for technical assistance. This work was funded by the Cooperative Research Centre for Discovery of Genes for Common Human Diseases and Cerylid Biosciences Pty Ltd. The CRC for Discovery of Genes for Common Human Diseases is established and supported by the Australian Government's Cooperative Research Centres Program. HC is a recipient of the McGill Fellowship of the Arthritis Foundation of Australia.

\section{Authors' affiliations}

J Stankovich, M M Sale, H M Cooley, A Reilly, J L Dickinson, G Jones, Menzies Centre for Population Health Research, University of Tasmania, GPO Box 252-23, Hobart, Tasmania, 7001, Australia M Bahlo, The Walter and Eliza Hall Institute of Medical Research, Royal Melbourne Hospital, Victoria, 3050, Australia

\section{REFERENCES}

1 March LM, Bachmeier CJ. Economics of osteoarthritis: a global perspective. Baillieres Clin Rheumatol 1997;1 1:817-34.

2 Jones G, Cooley HM, Bellamy N. A cross sectional study of the association between Heberden's nodes, radiographic osteoarthritis of the hands, grip strength, disability and pain. Osteoarthritis Cartilage 2001;9:606-1 1

3 Lawrence JS, Bremner JM, Biers F. Osteoarthritis. Prevalence in the population and relationship between symptoms and $x$ ray changes. Ann Rheum Dis 1966:25:1-24.

4 Hart DJ, Spector TD, Brown P, Wilson P, Doyle DV, Silman AJ. Clinical signs of early osteoarthritis: reproducibility and relation to $x$ ray changes in 541 women in the general population. Ann Rheum Dis 1991;50:467-70

5 Arden NK, Nevitt MC, Lane NE, Gore R, Hochberg MC, Scott JC, et al. Osteoarthritis and risk of falls, rates of bone loss, and osteoporotic fractures. Arthritis Rheum 1999:42:1378-85.

6 Plato CC, Norris AH. Osteoarthritis of the hand: age specific joint digit prevalence rates. Am J Epidemiol 1979;109:169-80.

7 Loughlin J. Genetic epidemiology of primary osteoarthritis. Curr Opin Rheumatol 2001;13:111-16.

8 Spector TD, Cicuttini F, Baker J, Loughlin J, Hart D. Genetic influences on osteoarthritis in women: a twin study. BM 1996:312:940-4.

9 Bijkerk C, Houwing-Duistermaat JJ, Valkenburg HA, Meulenbelt I, Hofman A, Breedveld FC, et al. Heritabilities of radiologic osteoarthritis in peripheral joints and of disc degeneration of the spine. Arthritis Rheum 1999;42:1729-35.

10 Ingvarsson T, Stefansson SE, Hallgrimsdottir IB, Frigge ML, Jonsson $H$, Jr., Gulcher J, et al. The inheritance of hip osteoarthritis in Iceland. Arthritis Rheum 2000:43:2785-92.

11 Kaprio J, Kujala UM, Peltonen L, Koskenvuo M. Genetic liability to osteoarthritis may be greater in women than men. BN 1996;313:212.

12 Felson DT, Couropmitree NN, Chaisson CE, Hannan MT, Zhang Y, McAlindon TE, et al. Evidence for a mendelian gene in a segregation analysis of generalized radiographic osteoarthritis. Arthritis Rheum 1998:41:1064-71.

13 Wright GD, Hughes AE, Regan M, Doherty M. Association of two loci on chromosome $2 \mathrm{q}$ with nodal osteoarthritis. Ann Rheum Dis 1996;55:317-19

14 Leppävuori J, Kujala U, Kinnunen J, Kaprio J, Nissilä M, Heliövaara M, et al. Genome scan for predisposing loci for distal interphalangeal joint osteoarthritis: evidence for a locus on 2q. Am J Hum Genet 1999;65:1060-7

15 Loughlin J, Mustafa Z, Smith A, Irven C, Carr AJ, Clipsham K, et al Linkage analysis of chromosome $2 q$ in osteoarthritis. Rheumatology 2000;39:377-81

16 Australian Bureau of Statistics. 1998 Population, Tasmania. Catalogue No 3235.6. Canberra: Commonwealth Government of Australia, 1998

17 Ad'Hiah AH, Mitchell J, Papiha SS. Alleles of complement components C4, C3, C2 and BR in the populations of Tasmania and northeast England. Gene Geography 1996;10:93-103.

18 Altman RD, Hochberg M, Murphy WA, Wolfe F, Lequesne M. Atlas of individual radiographic features in osteoarthritis. Osteoarthritis Cartilage 1995:3:A3-A70.

19 O'Connell JR, Weeks DE. PedCheck: A program for identification of genotype incompatibilites in linkage analysis. Am J Hum Genet 1998;63:259-266

20 Kellgren JH, Lawrence JS. Radiological assessment of osteoarthritis. Ann Rheum Dis 1957;16:494-501.

21 Kruglyak L, Daly M, Reeve-Dale MP, Lander ES. Parametric and non-parametric linkage analysis: a unified multipoint approach. Am J Hum Genet 1996;58:1347-63.

22 Kruglyak L, Lander ES. Faster multipoint linkage analysis using Fourier transforms. J Comput Biol 1998:5: 1-7.

23 Kong A, Cox NJ. Allele sharing methods: LOD scores and accurate linkage tests. Am J Hum Genet 1997;61:1179-88.

24 Cottingham RW Jr, Idury RM, Schaffer AA. Faster sequential genetic linkage computations. Am J Hum Genet 1993;53:252-63.

25 Almasy L, Blangero J. Multipoint quantitative trait linkage analysis in general pedigrees. Am J Hum Genet 1998;62:1198-211.

26 Blangero J, Williams JT, Almasy L. Variance component methods for detecting complex trait loci. Adv Genet 2001;42:151-81.

27 Williams JT, Blangero J. Power of variance component linkage analysis to detect quantitative trait loci. Ann Hum Genet 1999;63:545-63.

28 Rijsdijk FV, Hewitt JK, Sham PC. Analytic power calculation for QTL linkage analysis of small pedigrees. Eur J Hum Genet 2001;9:335-40 